\title{
Slowdown of light due to exciton-polariton propagation in $\mathrm{ZnO}$
}

Shula Chen, Weimin Chen and Irina Boyanova

\section{Linköping University Post Print}

N.B.: When citing this work, cite the original article.

Original Publication:

Shula Chen, Weimin Chen and Irina Boyanova, Slowdown of light due to exciton-polariton propagation in ZnO, 2011, Physical Review B. Condensed Matter and Materials Physics, (83), 24, 245212.

http://dx.doi.org/10.1103/PhysRevB.83.245212

Copyright: American Physical Society http://www.aps.org/

Postprint available at: Linköping University Electronic Press http://urn.kb.se/resolve?urn=urn:nbn:se:liu:diva-69847 


\title{
Slowdown of light due to exciton-polariton propagation in $\mathrm{ZnO}$
}

\author{
S. L. Chen, W. M. Chen, and I. A. Buyanova* \\ Department of Physics, Chemistry and Biology, Linköping University, 58183 Linköping, Sweden \\ (Received 1 February 2011; revised manuscript received 15 April 2011; published 30 June 2011)
}

\begin{abstract}
By employing time-of-flight spectroscopy, the group velocity of light propagating through bulk $\mathrm{ZnO}$ is demonstrated to dramatically decrease down to $2044 \mathrm{~km} / \mathrm{s}$ when photon energy approaches the absorption edge of the material. The magnitude of this decrease is found to depend on light polarization. It is concluded that even though the slowdown is observed in the vicinity of donor bound exciton (BX) resonances, the effect is chiefly governed by dispersion of free exciton (FX) polaritons that propagate coherently via ballistic transport. Based on the experimentally determined spectral dependence of the polariton group velocity, the polariton dispersion is accurately determined.
\end{abstract}

DOI: 10.1103/PhysRevB.83.245212

PACS number(s): 78.66.Hf, 78.47.D-

\section{INTRODUCTION}

There has been substantial fundamental and technological interest $\mathrm{t}^{1-5}$ to control and slow down light propagation in dispersive materials attributable to its great potential for a variety of innovative applications, e.g., in all optical communication networks, novel acousto-optical devices, quantum information storage and processing, etc. For example, it was shown ${ }^{6}$ that slowing the group velocity of a laser pulse down to the speed of sound in solids leads to enhanced acousto-optical effects, which is promising for applications in quantum optics for effective wave mixing and amplification. Implementation of controllable slow-light delays ${ }^{7,8}$ in photonic-crystal waveguides and fibers enables realization of all-optical buffers and variable optical delays. Moreover, laser pulses have even been brought to a halt in opaque atomic medium, which allowed storage of coherent optical information for up to 1 ms. ${ }^{9,10}$ This represents an important step toward quantum information storage and processing. In semiconductor materials the concept of slow light can be realized by utilizing singularities of dielectric function in the vicinity of excitonic resonances and exciton-photon coupling that leads to polariton formation. By using time-of-flight spectroscopy, a decrease in light group velocity owing to polariton dispersion has indeed been experimentally demonstrated for a variety of bulk semiconductors and related low-dimensional structures including bulk $\mathrm{CdSe},{ }^{11} \mathrm{GaP},{ }^{12} \mathrm{CuCl},{ }^{13} \mathrm{GaN},{ }^{14} \mathrm{CdZnTe},{ }^{15}$ and GaAs quantum wells. ${ }^{16}$

In recent years $\mathrm{ZnO}$ has been recognized to be among the most promising semiconductor materials for optical and electronic applications. As a wide bandgap semiconductor with a direct bandgap and large exciton-binding energy of about $60 \mathrm{meV}, \mathrm{ZnO}$ is a key candidate not only for biodetection and transparent electronics but also in optical communications, high-density data storage systems, as well as for short wavelength light emitters including lasers. Moreover light-matter interaction effects, such as polariton formation, are known ${ }^{17-19}$ to be exceptionally strong in $\mathrm{ZnO}$, facilitated by the polar-bonding character and large polarizability of $\mathrm{ZnO}$ matrix. Therefore polaritonic effects are foreseen to contribute to novel fascinating applications of this material, e.g., in polariton lasers operating at room temperature. ${ }^{20}$

In principle the polariton formation should also substantially affect group velocity of light propagating through $\mathrm{ZnO}$ media. The first experimental verification that the light can indeed be slowed down in $\mathrm{ZnO}$ was recently obtained from time-resolved photoluminescence (PL) studies of donor bound exciton (BX) emission. ${ }^{21}$ It was found that time required for transfer of the emitted photons from the $\mathrm{BX}$ recombination through a $1-\mathrm{mm} \mathrm{ZnO}$ crystal is rather long, i.e., reaching $200 \mathrm{ps}$, which was attributed to the dispersive propagation of the BX polaritons. However, analysis of the results was complicated by the fact that the delay of the detected BX emission was partly related to delayed formation of the BX following expansion of electron-hole plasma through the crystal from the front surface of the sample where it was initially excited.

In the present study we avoid this complication by investigating propagation of laser pulses instead. We show that light experiences dramatic slowdown when photon energies approach the absorption edge of $\mathrm{ZnO}$, to a degree determined by light polarization. This effect is analyzed in the framework of polariton propagation. It is concluded that even though the slowdown is observed in the vicinity of donor BX resonances, the main contribution to the effect in fact arises from free exciton (FX) polaritons that propagate coherently via ballistic transport. Based on the experimentally determined spectral dependence of the polariton group velocity, the polariton dispersion is accurately determined.

\section{SAMPLES AND METHODS}

Light propagation was studied by time-of-flight spectroscopy using commercially available c-plane $\mathrm{ZnO}$ substrates from Cermet, Inc. and Denpa Co. with thicknesses of 0.25 and $0.55 \mathrm{~mm}$, respectively. To reduce light absorption in the vicinity of excitonic resonances, some of the Denpa $\mathrm{ZnO}$ substrates were mechanically polished down to the thickness of $0.13 \mathrm{~mm}$. Effects of polarization of light on its group velocity in the wurtzite matrix were evaluated by performing time-of-flight measurements for the following relative orientations of the light wave vector $\boldsymbol{k}$, the electrical field vector $\boldsymbol{E}$, and the principal axis $\boldsymbol{c}$ of wurtzite $\mathrm{ZnO}$ : (i) $\boldsymbol{k} \| \boldsymbol{c}, \boldsymbol{E} \perp \boldsymbol{c}(\alpha$-polarization); (ii) $\boldsymbol{k} \perp \boldsymbol{c}, \boldsymbol{E} \perp \boldsymbol{c}(\sigma$-polarization); and (iii) $\boldsymbol{k} \perp \boldsymbol{c}, \boldsymbol{E} \| \boldsymbol{c}$ ( $\pi$-polarization). In the two latter cases, a 1-mm-thick sample cut from the Denpa ZnO substrate was used. 

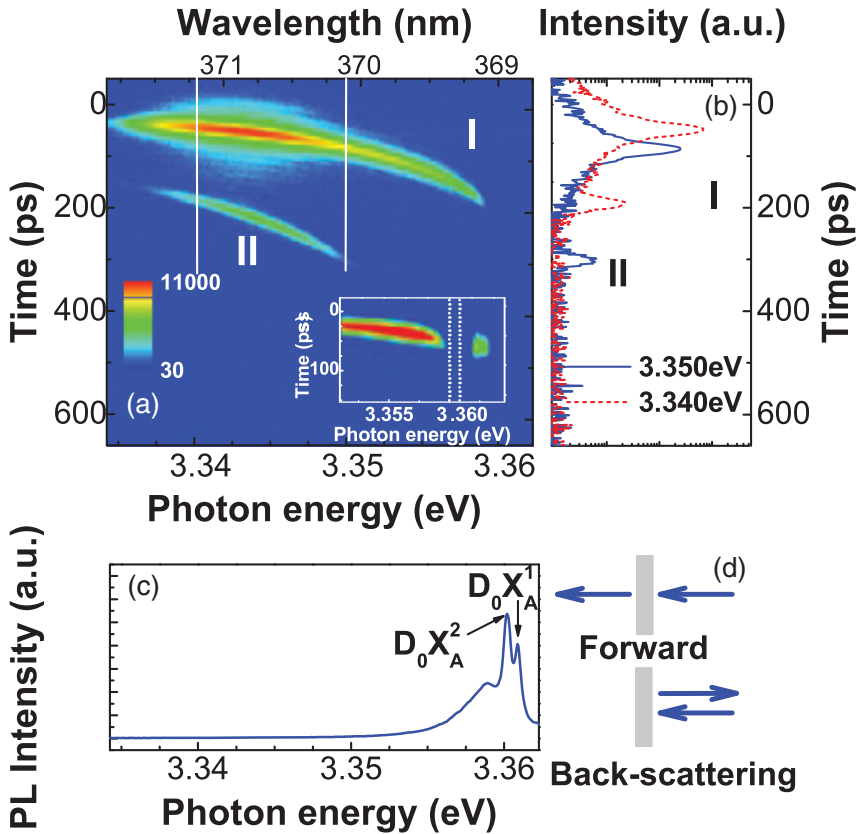

FIG. 1. (Color online) (a) Transient spectra of a 3.351-eV laser pulse transmitted through the $0.55-\mathrm{mm} \mathrm{ZnO}$ sample in the $\alpha$ measurement geometry $(\boldsymbol{k} \| \boldsymbol{c}, \boldsymbol{E} \perp \boldsymbol{c})$. The light intensity is displayed in the logarithmic scale. The stronger signal denoted as "I" corresponds to the main laser pulse, whereas the weaker component "II" is attributed to the light pulse that has experienced two internal reflections at the sample surfaces before exiting the sample. (b) Temporal profiles of the transmitted light with energies as indicated by the solid lines in (a). (c) Typical PL spectrum recorded in the back-scattering geometry. All measurements were performed at $4 \mathrm{~K}$. (d) A schematic illustration of the measurement geometries employed in the time-of-flight and PL measurements. The inset in (a) shows a close up of the transient spectra within the $D_{0} X_{\mathrm{A}}$ spectral region. The dotted lines in the inset indicate the spectral positions of the $D_{0} X_{\mathrm{A}}^{1}$ and $D_{0} X_{\mathrm{A}}^{2}$ resonances. The spectra were measured for the 0.13 -mm-thick $\mathrm{ZnO}$.

Time-resolved optical measurements were carried out at temperatures of $4-150 \mathrm{~K}$ using a tunable Ti:sapphire femtosecond-pulsed laser with a repetition frequency of $76 \mathrm{MHz}$ and duration of about $150 \mathrm{fs}$. Second-harmonic laser pulses tuned below the FX but in the vicinity of BX energies (3.326-3.361 eV) were used as a light source during time-of-flight measurements. This allowed us to solely study propagation of the laser light and to avoid PL excitation. The transmitted light was detected in the forward geometry with light propagating along the normal to the sample surface [Fig. 1(d)]. The time origin was calibrated by carefully removing the sample out of the beam. Typical laser power was around $10 \mathrm{~W} / \mathrm{cm}^{2}$. PL measurements were performed in the back-scattering geometry under excitation by third-harmonic laser pulses with the photon energy of $4.66 \mathrm{eV}$. The transmitted laser light and PL were detected by a Hamamatsu streak camera combined with a $0.5-\mathrm{m}$ grating monochromator. Time resolution of the whole system was better than 2 ps.

\section{EXPERIMENTAL RESULTS}

Figure 1(a) shows a representative image of a laser pulse, which was initially tuned to $3.351 \mathrm{eV}$, after it was transmitted through a $0.55-\mathrm{mm}$-thick Cermet $\mathrm{ZnO}$ sample in the $\alpha$-measurement geometry $(\boldsymbol{k} \| \boldsymbol{c}, \boldsymbol{E} \perp \boldsymbol{c})$. Several important features can be noticed. First of all, the light could only be transmitted when photon energies are below $3.360 \mathrm{eV}$, which indicates strong near-band-edge absorption at the higher energies. [After decreasing thickness of the sample down to $0.13 \mathrm{~mm}$, light propagation can be studied even for photon energies slightly above the $D^{0} X_{\mathrm{A}}$ resonances as shown in the inset of Fig. 1(a)]. Second, the image (i.e., streak) of the transmitted light is strongly bent toward the absorption edge. This means that the time required for light propagation through the sample (to be referred further as time delay) critically depends on photon energy and increases when the photon energy approaches the region of near-band-edge excitonic absorption. For example the time delay for the main component (denoted as "I") increases from about $49 \mathrm{ps}$ for the $3.335 \mathrm{eV}$ photons to up to $217 \mathrm{ps}$ for the $3.360 \mathrm{eV}$ photons. This dependence can also be seen from Fig. 1(b), which shows time profiles of the transmitted light for energies as indicated by the solid lines in Fig. 1(a). Moreover, in addition to the intense signal from the main laser pulse, the image contains a much weaker signal denoted as "II" in Figs. 1(a) and 1(b). The corresponding time delay at any wavelength is about three times longer than that for the main transmitted pulse, which results in stronger bending of the streak. We attribute this feature to the light pulse which has experienced two internal reflections at the sample surfaces (which act as a Fabry-Perot cavity) before exiting the sample, i.e., propagated inside the sample in forward-backward-forward directions. Comparison with the reference PL spectra [Fig. 1(c)] shows that the observed slowdown occurs in the vicinity of donor $\mathrm{BX}$ resonances denoted as $D^{0} X_{\mathrm{A}}^{1}$ and $D^{0} X_{\mathrm{A}}{ }^{2}$ in Fig. 1(c).

The slowdown of photons with energies in the vicinity of the absorption edge is also observed in the $\pi$ - (i.e., when $\boldsymbol{k} \perp \boldsymbol{c}$, $\boldsymbol{E} \| \boldsymbol{c}$ ) and $\sigma$ - (i.e., when $\boldsymbol{k} \perp \boldsymbol{c}, \boldsymbol{E} \perp \boldsymbol{c}$ ) geometries, as shown in Figs. 2(a) and 2(b), respectively. In these cases, however, only the main transmitted pulse is observed, likely due to poorer quality of the surfaces after cutting the sample. In the $\sigma$-geometry light propagation remains basically identical to that observed in the $\alpha$-geometry discussed previously. On the other hand it is significantly modified when the light wave has $\pi$-polarization. Under this condition the $\mathrm{ZnO}$ samples become transparent in the regions of the $D^{0} X_{\mathrm{A}}^{1}$ and $D^{0} X_{\mathrm{A}}^{2}$ absorption, and a blue shift of the absorption edge up to approximately $3.375 \mathrm{eV}$ is observed. With increasing power of the laser light up to $800 \mathrm{~W} / \mathrm{cm}^{2}$, the transmitted signal can be detected within the spectral range of 3.3771-3.3810 eV (i.e., above the $3.375-\mathrm{eV}$ resonance) even for the $1-\mathrm{mm}$-thick $\mathrm{ZnO}$ [see the inset of Fig. 2(a)]. It is also noticeable that the bending of the streak toward the absorption edge is by far less pronounced for the $\pi$-polarization, meaning shorter time delays.

The observed dependence of the light absorption on light polarization can be understood in light of selection rules for optical transitions in the wurtzite matrix. Indeed, crystal-field and spin-orbit interactions split valence band $(\mathrm{VB})$ of $\mathrm{ZnO}$ into the so-called A-, B-, and C-subbands. Assuming an inverse ordering of the VB states, ${ }^{22-24}$ i.e., $\mathrm{A}\left(\Gamma_{7}\right), \mathrm{B}\left(\Gamma_{9}\right)$, and $\mathrm{C}\left(\Gamma_{7}\right)$ states, symmetries of the corresponding excitonic states in $\mathrm{ZnO}$ are $\Gamma_{7} \otimes \Gamma_{7}=\Gamma_{1} \otimes \Gamma_{2} \otimes \Gamma_{5}, \Gamma_{7} \otimes \Gamma_{9}=\Gamma_{6} \otimes \Gamma_{5}$, 


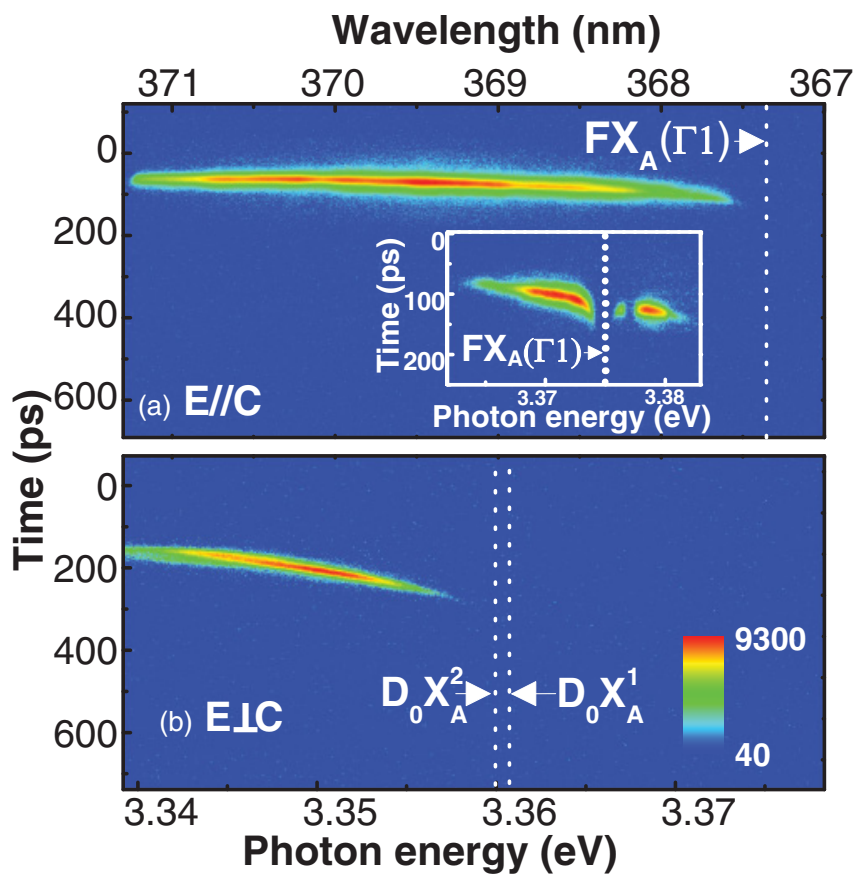

FIG. 2. (Color online) Transient spectra of a 3.358-eV laser pulse with the (a) $\pi$ - and (b) $\sigma$-polarization after transmission through the 1-mm-thick $\mathrm{ZnO}$ sample. The light intensity is displayed in the logarithmic scale. The dotted lines indicate positions of the specified excitonic resonances. The employed laser power was around $10 \mathrm{~W} / \mathrm{cm}^{2}$. The inset in (a) shows a close up of the transient spectra in the vicinity of the $3.375-\mathrm{eV}$ resonance. The spectra were measured after increasing the laser power to $800 \mathrm{~W} / \mathrm{cm}^{2}$.

and $\Gamma_{7} \otimes \Gamma_{7}=\Gamma_{1} \otimes \Gamma_{2} \otimes \Gamma_{5}$ for the A-, B-, and C-excitons, respectively. From group theory considerations the only dipole-allowed transitions are $\Gamma_{5}$ for $\boldsymbol{E} \perp \boldsymbol{c}$ and $\Gamma_{1}$ for $\boldsymbol{E} \| \boldsymbol{c}$ polarizations. Moreover, oscillator strengths of the A and $\mathrm{B} \Gamma_{5}$ excitons are significantly larger than that for the $\mathrm{C}$ $\Gamma_{5}$. On the other hand the $\mathrm{A} \Gamma_{1}$ excitons are spin-forbidden and consequently have low oscillator strength, whereas the $\mathrm{C}$ $\Gamma_{1}$ excitons are spin-allowed. ${ }^{24}$ Therefore, optical-absorption transitions related to the upper A- and B-VB subbands (and the associated $D^{0} X$ resonances) are the most pronounced ones in the $\alpha$ - and $\sigma$-geometries, consistent with our experimental data. In contrast the $\mathrm{A} \Gamma_{1}$ resonance, which defines the $\mathrm{ZnO}$ absorption edge for the $\boldsymbol{E} \| \boldsymbol{c}$ light polarizations, is rather weak. Spectral positions of the relevant excitonic resonances are indicated by the dotted lines in Figs. 2 and 3. From the inset in Fig. 2(a) one may also notice the presence of an even weaker resonance located at $\sim 2 \mathrm{meV}$ above the $\mathrm{A} \Gamma_{1}$. The spectral position of this resonance corresponds to that of a longitudinal $\mathrm{A} \Gamma_{5 \mathrm{~L}}$ exciton, ${ }^{23}$ which may become weakly visible when $\boldsymbol{E} \| \boldsymbol{c}$ because of, e.g., coupling with A $\Gamma_{1}$ or imperfect optical alignment. This resonance will, however, not be further considered as it has a very low oscillator strength and does not affect time delays of the transmitted light.

The results from the time-of-flight measurements of all investigated samples obtained in three measurement geometries are summarized in Fig. 3, where symbols denote the experimentally measured delay times for the $\mathrm{ZnO}$ samples with the specified thicknesses. Data denoted by dots represent

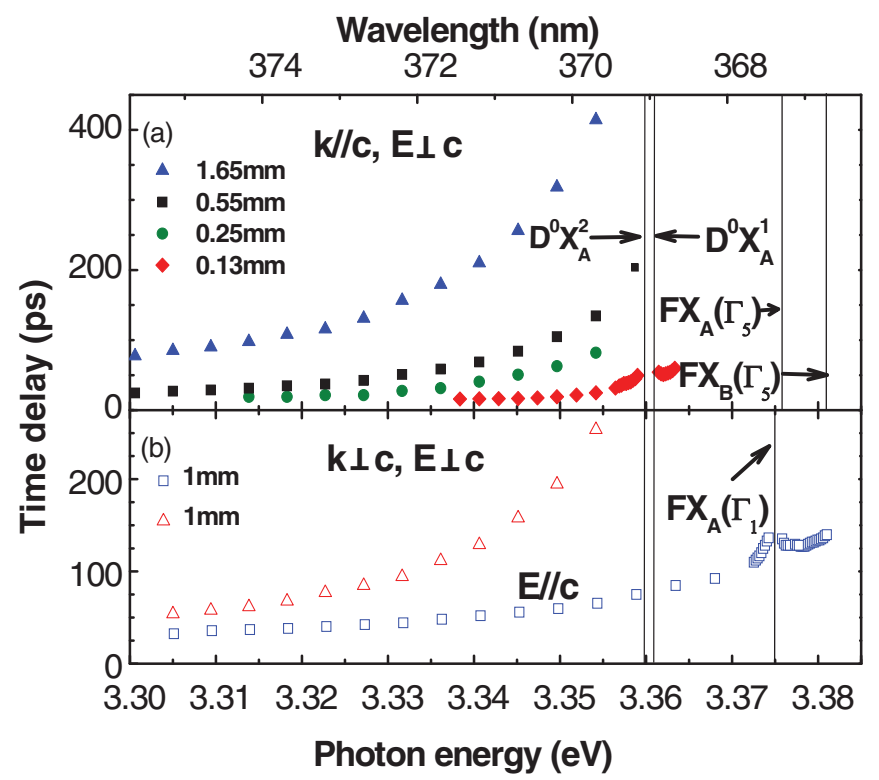

FIG. 3. (Color online) Time delays of laser pulses caused by propagation through the $\mathrm{ZnO}$ media with the specified thickness for (a) $\boldsymbol{k} \| \boldsymbol{c}$ and (b) $\boldsymbol{k} \perp \boldsymbol{c}$. The data points attributed to the 1.65 -mm-thick $\mathrm{ZnO}$ represent time delays for the signal "II" shown in Fig. 1, i.e., for a laser pulse that has undergone the forward-backward-forward propagation inside the $0.55-\mathrm{mm}$ sample. The solid lines represent spectral positions of the marked excitonic resonances.

the time delays in the Cermet $\mathrm{ZnO}$, whereas all other data were obtained from the Denpa samples. Clearly the slowdown effect is common in $\mathrm{ZnO}$ and relies on the presence of excitonic resonances as a magnitude of the effect largely depends on light polarization.

Light propagation also remains dispersive at elevated temperatures. This is illustrated in Fig. 4 where spectral dependences of the delay time measured at different temperatures in

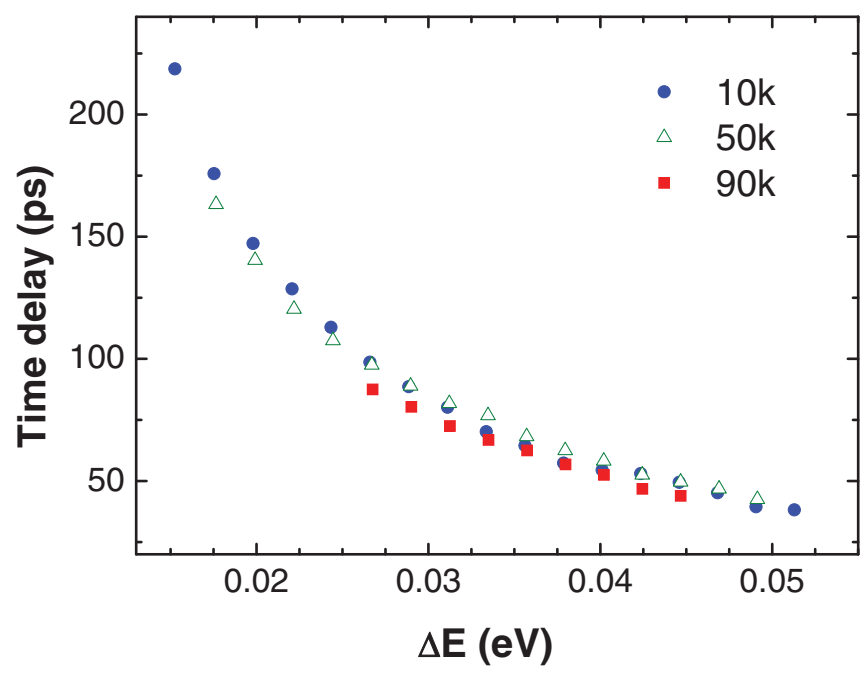

FIG. 4. (Color online) Time delays of the transmitted laser pulse measured from the $0.55-\mathrm{mm}$-thick $\mathrm{ZnO}$ at the specified temperatures. The data are plotted as a function of energy distance $\Delta \mathrm{E}$ to the $\mathrm{FX}_{\mathrm{A}}\left(\Gamma_{5}\right)$ exciton to account for a temperature-induced shift of the $\mathrm{ZnO}$ bandgap. 
the $\alpha$-geometry are shown. The data are plotted as a function of energy distance to the $\mathrm{FX}_{\mathrm{A}}\left(\Gamma_{5}\right)$ exciton to account for a temperature-induced shift of the $\mathrm{ZnO}$ bandgap and, therefore, of the spectral position of the $\operatorname{FX}_{\mathrm{A}}\left(\Gamma_{5}\right)$. The latter was determined based on the performed temperature-dependent PL studies. The measurements were unfortunately limited to temperatures up to $90 \mathrm{~K}$, as strong broadening of the absorption edge at higher temperatures hampered light transmission close to the excitonic resonances.

\section{DISCISSION}

\section{A. Mechanism for light propagation}

Before discussing the origin of the observed slowdown of light in $\mathrm{ZnO}$ in the vicinity of the excitonic resonances, let us first elucidate the mechanism responsible for light propagation. In a dielectric medium, light propagation could be either ballistic ${ }^{11,12,14}$ or diffusive ${ }^{14}$ depending on concentration and scattering cross-section of scattering centers in the material. Both mechanisms could, in principle, account for the resonant decrease of light velocity in the regions of excitonic resonances. ${ }^{14}$ However while the diffusive propagation features a broad outgoing angle of the transmitted light, a narrow angular distribution is expected in the ballistic regime. This allows us to discriminate between these mechanisms by measuring the angular distribution of light intensity after transmission through the sample. Representative results are shown in Figs. 5(a) and 5(b) for the Denpa and Cermet ZnO, respectively. Only a very weak divergence of the transmitted laser light, which is independent of the photon energy, is observed suggesting that the light propagation in the investigated samples is predominantly ballistic. This conclusion is further supported by the observation of the "reflection" streak [signal "II" in Figs. 1(a) and 1(b)], i.e., the transmitted laser pulse that experienced two internal reflections. This finding indicates weak scattering of the laser light, which is characteristic for the ballistic regime. We note, however, that the "reflection" signal was not detected in the Cermet $\mathrm{ZnO}$, which may imply a weak contribution of the diffusive component.

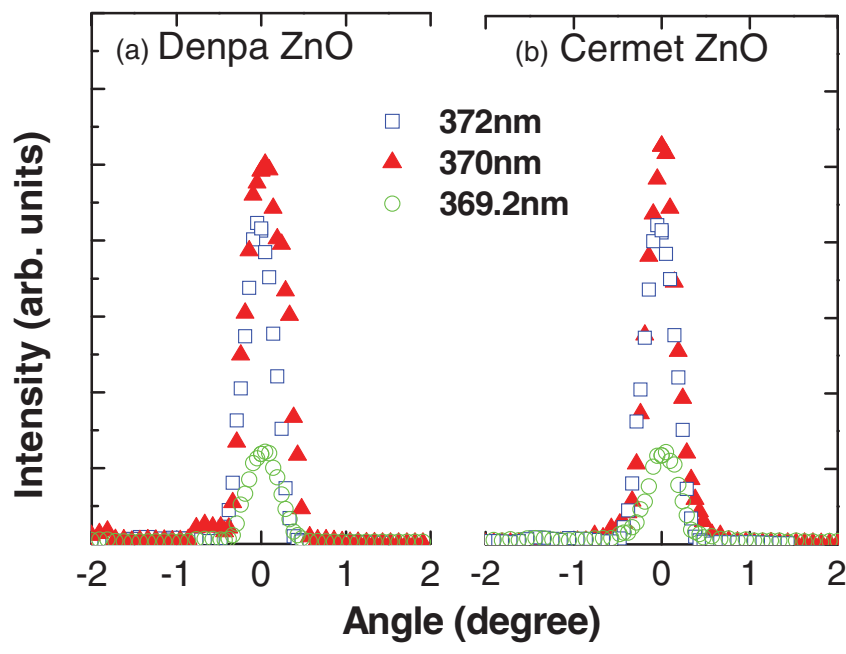

FIG. 5. (Color online) Angular distributions of the transmitted light, with the wavelengths of 369.2, 370 and $372 \mathrm{~nm}$, through the (a) Denpa and (b) Cermet $\mathrm{ZnO}$.
Under conditions of the ballistic transport, light wave will propagate coherently with a group velocity $v_{g}$ defined as

$$
v_{g}(E)=\frac{1}{\hbar} \frac{d E}{d K} .
$$

Since the time required for light propagation in this regime linearly depends on the sample thickness $L$, the group velocity and its dispersion can be experimentally determined by $v_{g}=$ $L / T_{d}$ from the measured time delay $T_{d}$. The obtained spectral dependence of $v_{g}$ is shown in Fig. 6. It provides evidence that the light velocity in $\mathrm{ZnO}$ dramatically decreases down to $\sim 2 \times$ $10^{6} \mathrm{~m} / \mathrm{s}$, i.e., 1500 times slower than the speed of light in vacuum, when approaching excitonic resonances. Moreover, under these conditions the group velocity can be controlled by changing polarization of the propagating light, e.g., from $2 \times 10^{6} \mathrm{~m} / \mathrm{s}$ when $\boldsymbol{E} \perp \boldsymbol{c}$ to $1.1 \times 10^{7} \mathrm{~m} / \mathrm{s}$ when $\boldsymbol{E} \| \boldsymbol{c}$ for the 3.365-eV photons.

\section{B. Origin of the slowdown effect}

The observed spectral dependence of the group velocity can be understood by taking into account exciton-photon coupling, which leads to the formation of a mixed-mode state called exciton-polariton that is now a true eigenstate of the system. Within the polariton framework the light wave entering the sample is converted into the polariton wave which then propagates through the media with the group velocity determined by the polariton dispersion. ${ }^{14,17,18}$ In the case of several excitonic resonances the dispersion of the exciton-polaritons neglecting the spatial dispersion can be expressed as

$$
\begin{aligned}
\frac{\hbar^{2} c^{2} K^{2}}{E^{2}}= & \varepsilon_{\infty}+\sum_{i=D^{0} X_{A}^{i}} \frac{f_{i} E_{0 i}^{2}}{E_{0 i}^{2}-E^{2}-i E \hbar \Gamma_{i}} \\
& +\sum_{j=A, B, C} \frac{f_{j} E_{0 j}^{2}}{E_{0 j}^{2}-E^{2}-i E \hbar \Gamma_{j}} .
\end{aligned}
$$

Here $\varepsilon_{\infty}$ is the background dielectric constant. The second and the third term represent contributions from the $D^{0} X$ and FX resonances, respectively, where the summation should be done over all optically active states. $E_{0}, f$, and $\Gamma$ are the resonance energy, oscillator strength, and the damping constant (considered to be wavevector $\mathrm{K}$ independent), respectively. The subscript $i(j)$ denotes the association of these parameters to the specific $D^{0} X(\mathrm{FX})$ resonance. We would like to note that even though the polariton concept in $\mathrm{ZnO}$ has usually been applied to FX polaritons, contributions of the BXs were also included in Eq. (2) for completeness of the analysis. ${ }^{14}$ Equations (1) and (2) can be used to simulate spectral dependence of the polariton-group velocity. The corresponding results are shown by the solid (black dashed) lines in Fig. 6 for the Denpa (Cermet) $\mathrm{ZnO}$ using the parameters given in Table I. The background dielectric constants $\varepsilon_{\infty}$ for $\boldsymbol{E} \perp \boldsymbol{c}$ and $\boldsymbol{E} \| \boldsymbol{c}$ light polarizations were set to 6.69 and 7.47 , respectively. ${ }^{25}$ The damping term $\hbar \Gamma_{j}$ for all resonances was assumed to be $0.5 \mathrm{meV}$, which is of the order of the PL linewidth. For the Denpa $\mathrm{ZnO}$ only $D^{0} X_{\mathrm{A}}^{1}$ and $D^{0} X_{\mathrm{A}}^{2}$ resonances were considered, and the ratio between their oscillator strengths was estimated based on the measured ratio between the 


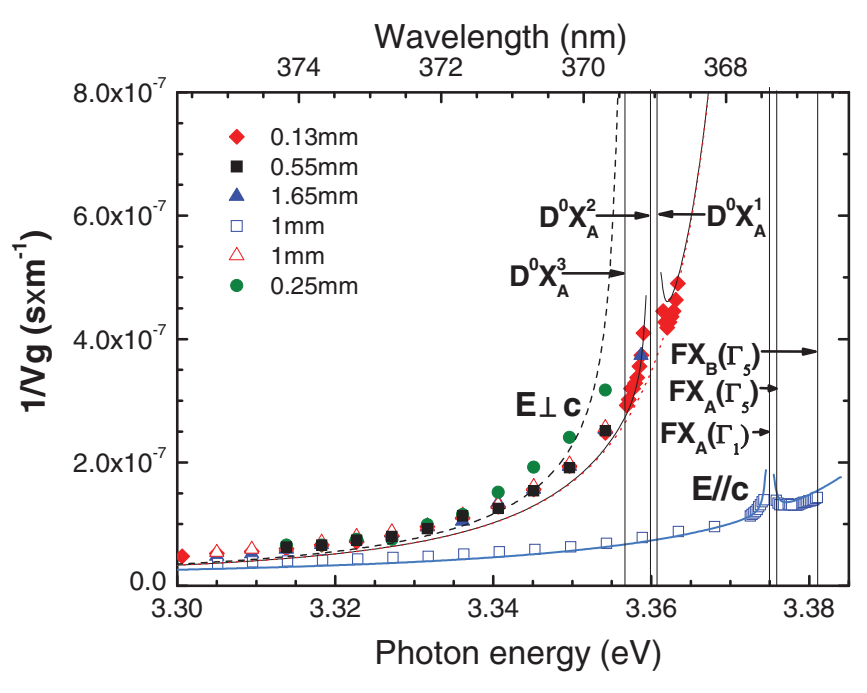

FIG. 6. (Color online) Spectral dependence of the reverse group velocity. The symbols represent experimental data, and the curves are the simulation results using Eq. (2) with the parameters listed in Table I. The solid (dashed) curves represent a best fit for the Denpa (Cermet) $\mathrm{ZnO}$. The red dotted curve represents results of the simulations performed by omitting contributions of all $D^{0} X$ resonances. Note that the simulated time delays within the spectral range of the strong absorption from the $D^{0} X$ and $\mathrm{FX}_{\mathrm{A}}\left(\Gamma_{1}\right)$ resonances are not shown here for clarity. The vertical lines denote the spectral positions of the excitonic resonances as specified.

corresponding PL intensities. For the Cermet sample, another resonance at $3.3567 \mathrm{eV}\left(D^{0} X_{\mathrm{A}}^{3}\right)$ observed in both transmission and PL spectra was also included. The spectral positions of the FX resonances were taken from Refs. 17 and 26, while their $f_{j}$ values were chosen based on the reported values ${ }^{17}$ of the corresponding longitudinal-transverse splitting. These values are consistent with the measured spectral positions of the $D^{0} X_{\mathrm{A}}$ and FX-PL lines in the investigated samples, which are in excellent agreement with the reported values for bulk strain-free $\mathrm{ZnO}{ }^{26}$ The oscillator strengths for the $D^{0} X$ resonances were treated as fitting parameters. The best fit to the experimental data for the $\boldsymbol{E} \perp \boldsymbol{c}$ light polarizations was obtained assuming $f\left(D^{0} X_{A}^{1}\right)=1.3 \times 10^{-5}$ and $f\left(D^{0} X_{A}^{2}\right)=$ $2.6 \times 10^{-5}$ for the Denpa $\mathrm{ZnO}$ (shown by the black solid line in Fig. 6), whereas higher oscillator strengths for these

TABLE I. The parameters of the excitonic resonances obtained from a best fit of Eqs. (1) and (2) to the experimental data.

\begin{tabular}{llll}
\hline \hline & & \multicolumn{2}{c}{ Oscillator strength, $\mathrm{f}$} \\
\cline { 3 - 4 } Transition & $\mathrm{E}_{0}(\mathrm{eV})$ & $\boldsymbol{E} \perp \mathbf{c}$ & $\boldsymbol{E} \| \boldsymbol{c}$ \\
\hline $\mathrm{FX}_{\mathrm{A}}(\Gamma 5)$ & 3.3758 & 0.0071 & 0 \\
$\mathrm{FX}_{\mathrm{B}}(\Gamma 5)$ & 3.3810 & 0.0404 & 0 \\
$\mathrm{FX}_{\mathrm{A}}(\Gamma 1)$ & 3.3750 & 0 & 0.0009 \\
$\mathrm{FX}_{\mathrm{C}}(\Gamma 1)$ & 3.4198 & 0 & 0.0727 \\
$D_{0} X_{\mathrm{A}}^{1}$ (Denpa) & 3.3608 & $1.3 \times 10^{-5}$ & 0 \\
$D_{0} X_{\mathrm{A}}^{1}$ (Cermet) & 3.3608 & $8.8 \times 10^{-4}$ & 0 \\
$D_{0} X_{\mathrm{A}}^{2}$ (Denpa) & 3.3600 & $2.6 \times 10^{-5}$ & 0 \\
$D_{0} X_{\mathrm{A}}^{2}$ (Cermet) & 3.3600 & $8.8 \times 10^{-4}$ & 0 \\
$D_{0} X_{\mathrm{A}}^{3}$ (Cermet) & 3.3567 & $1.7 \times 10^{-3}$ & 0 \\
\hline \hline
\end{tabular}

resonances (see Table I) were required to reproduce the $v_{g}(E)$ dependence for the Cermet sample (shown by the black dashed line in Fig. 6).

In order to cross check whether the obtained fitting parameters are reasonable, we have applied them to estimate the concentrations of the related donor impurities. The oscillator strength of the $D^{0} X$ resonance depends on the donor concentration $N$ as described by ${ }^{27}$

$$
f=\frac{4 \pi N}{E_{0, D X}}\left(\frac{\hbar e\left|P_{c v}\right|}{m_{0} E_{0, D X}}\right)^{2}\left[\int \psi(r, 0) d r\right]^{2},
$$

where $P_{c v}$ is the momentum matrix element, $e$ is the electron charge, and $m_{0}$ is the free-electron mass. The exciton wavefunction $\psi(r, \rho)$ has the form ${ }^{14}$

$$
\psi(r, \rho)=\frac{1}{\pi R^{3 / 2} a_{B}^{3 / 2}} \exp (-r / R) \exp \left(-\rho / a_{B}\right) .
$$

Here $\rho$ denotes the relative electron-hole vector, $r$ is the position of the center of mass, $a_{B}$ is the Bohr radius $\left(a_{B}=2.34 \mathrm{~nm}\right),{ }^{28}$ and $R$ is the localization radius taken equal to $a_{B}$. Taking values of $P_{c v}$ from Ref. 29, the total residual donor concentration can be estimated to be about $2 \times$ $10^{15} \mathrm{~cm}^{-3}$ for the Denpa $\mathrm{ZnO}$, which is reasonable for the high-quality material. The value is higher for the Cermet sample, which is consistent with a higher position of the Fermi level such that the donor levels are readily occupied by electrons in dark in this material as revealed from our earlier electron spin-resonance studies. ${ }^{30}$ A higher concentration of residual impurities could also somewhat explain enhanced light scattering in this material.

The obtained excellent agreement between the experimental data and simulated spectral dependence of polariton-group velocity evidences that the observed decrease of the light velocity in the vicinity of the absorption edge is caused by the formation of exciton-polaritons and is determined by their dispersion. Furthermore the performed simulations allow us to elucidate contributions of different resonances to the slowdown effect. For the $\boldsymbol{E} \perp \boldsymbol{c}$ polarization the polariton-group velocity is shown to be mainly determined by the tail of the spectral dispersion within the lower branch of the $\mathrm{FX}_{\mathrm{A}}$ $\left(\Gamma_{5}\right)$ polaritons, whereas contributions of the $D^{0} X_{\mathrm{A}}$ polaritons become important only in close proximity of the corresponding resonances (i.e., within $\sim 10 \mathrm{meV}$ for Cermet $\mathrm{ZnO}$ ). This is obvious from the results of the simulations performed by omitting all $D^{0} X$ resonances (see the red dotted line in Fig. 6). The minor role of the $D^{0} X_{\mathrm{A}}$ polaritons could be attributed to the weaker oscillator strength of the $D^{0} X_{\mathrm{A}}$ excitons as compared with the $\mathrm{FX}_{\mathrm{A}}$ transitions for the $\boldsymbol{E} \perp \boldsymbol{c}$ polarization. Consistently the dominant effect responsible for the reduction of light velocity for the $\boldsymbol{E} \| \boldsymbol{c}$ polarization is the $\mathrm{FX}_{\mathrm{C}}\left(\Gamma_{1}\right)$ polariton formation.

Based on the obtained parameters for the $D^{0} X_{\mathrm{A}}$ resonances, we can also briefly discuss to what extent the polariton concept is adequate for the description of BXs in the investigated samples. The polariton framework is usually applied in the case of strong exciton-photon coupling, which is valid when $E_{0, i}^{2} f_{i} / E \Gamma \geqslant \varepsilon_{\infty} .{ }^{12}$ This condition is fulfilled for the Cermet $\mathrm{ZnO}$ with the higher concentration of residual donors where $E_{0, i}^{2} f_{i} / E \Gamma \sim 20$ (if using the combined oscillator strengths 


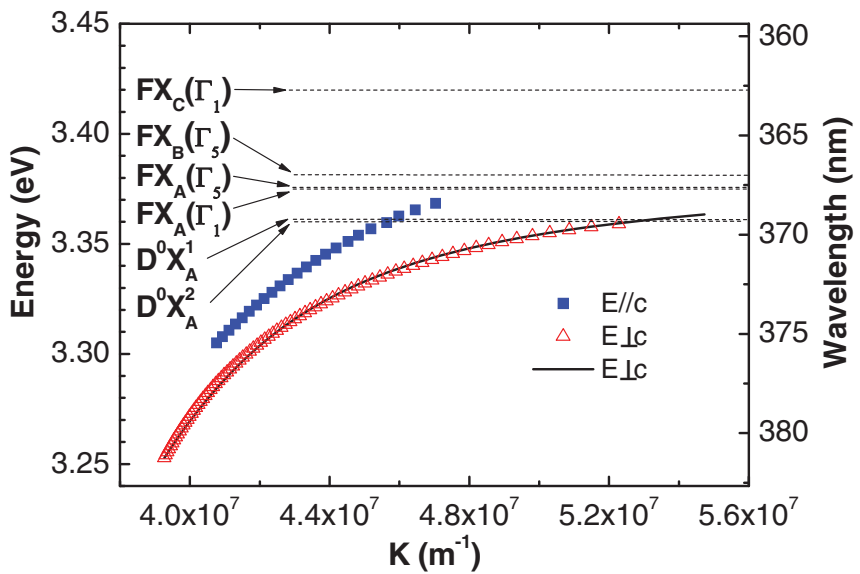

FIG. 7. (Color online) Polariton dispersion within the lower polariton branch, calculated based on the measured group velocity. The results of the calculations for $\boldsymbol{E} \perp \boldsymbol{c}$ and $\boldsymbol{E} \| \boldsymbol{c}$ light polarizations are shown by the open triangles and the filled squares, respectively. The horizontal dotted lines denote the spectral positions of the specified excitonic resonance. The open triangles (the solid line) for $\boldsymbol{E} \perp \boldsymbol{c}$ represent the polariton dispersion obtained taking into account (neglecting) data in the vicinity of the $D^{0} X_{\mathrm{A}}$ resonances.

of $D^{0} X_{\mathrm{A}}$ ). On the other hand light is only weakly coupled to the $D^{0} X_{\mathrm{A}}$ excitons in the Denpa $\mathrm{ZnO}$. However, even in the latter case the performed analysis using Eq. (2) (including the $D^{0} X_{\mathrm{A}}$ contributions) is believed to be relevant as the weak-coupling limit is an extreme case of the exciton-photon coupling. ${ }^{12,31,32}$

\section{Polariton dispersion}

Based on the experimentally determined spectral dependence of the polariton-group velocity, we can also reconstruct the polariton dispersion within the low polariton branch. Indeed from Eq. (1) the polariton dispersion could be derived as

$$
K(E)=K_{0}\left(E_{0}\right)+\frac{1}{\hbar} \int_{E_{0}}^{E} \frac{1}{\mathrm{v}_{\mathrm{g}}(E)} d E .
$$

Therefore the dispersion in the near-resonance spectral range can be obtained by integrating the experimentally measured $v_{g}(E)$ dependence (Fig. 6), provided that far from the resonance the polariton energy $\left(E_{0}\right)$ and the corresponding wavevector $\left(K_{0}\right)$ are known. The results of the calculations for $\boldsymbol{E} \perp \boldsymbol{c}$ and $\boldsymbol{E} \| \boldsymbol{c}$ light polarizations are shown in Fig. 7. Here we used $\mathrm{E}_{0, \perp}=3.25 \mathrm{eV}, \mathrm{K}_{0, \perp}=3.92 \times 10^{7} \mathrm{~m}^{-1}$, and $\mathrm{E}_{0, \|}=$ $3.30 \mathrm{eV}, \mathrm{K}_{0, \|}=4.07 \times 10^{7} \mathrm{~m}^{-1}$, based on reported values of the refractive index of $\mathrm{ZnO} .{ }^{33}$ The open triangles (the solid line) for $\boldsymbol{E} \perp \boldsymbol{c}$ represent the polariton dispersion obtained taking into account (neglecting) data in the vicinity of the $D^{0} X_{\mathrm{A}}$ resonances. The lower polariton branch exhibits a photon-like dispersion in the spectral region transparent to light, which becomes exciton-like when approaching the FX resonances, consistent with the expected dispersion for exciton-polaritons. It is also obvious that the dispersion is mainly determined by the photon coupling with the FXs.

\section{CONCLUSIONS}

In conclusion, we have employed the time-of-flight spectroscopy to study light propagation though the $\mathrm{ZnO}$ media. The group velocity of light is found to dramatically decrease down to $2044 \mathrm{~km} / \mathrm{s}$ when photon energies approach the absorption edge of the material. It is also demonstrated that the magnitude of this slowdown of light can be controlled by changing light polarization. The effect is explained within the polariton framework and is concluded to be mainly determined by the dispersion of FX polaritons, which propagate coherently via ballistic transport. Based on the experimentally obtained spectral dependence of the polariton-group velocity, the polariton dispersion within the lower polariton branch is accurately determined.

\section{ACKNOWLEDGMENT}

Financial support by the Swedish Research Council (Grant No. 621-2010-3971) is greatly appreciated. *iribu@ifm.liu.se

${ }^{1}$ M. D. Lukin and A. Imamoglu, Nature 413, 273 (2001).

${ }^{2}$ A. V. Turukhin, V. S. Sudarshanam, M. S. Shahriar, J. A. Musser, B. S. Ham, and P. R. Hemmer, Phys. Rev. Lett. 88, 023602 (2002).

${ }^{3}$ M. Bajcsy, A. S. Zibrov, and M. D. Lukin, Nature 426, 638 (2003).

${ }^{4}$ J. T. Mok and B. J. Eggleton, Science 433, 811 (2005).

${ }^{5}$ C. J. Chang-Hasnain and S. L. Chuang, J. Lightwave Technol. 24, 4642 (2006).

${ }^{6}$ A. Matsko, Y. Rostovtsev, H. Cummins, and M. Scully, Phys. Rev. Lett. 84, 5752 (2000).

${ }^{7}$ Y. A. Vlasov, M. O’Boyle, H. F. Hamann, and S. J. McNab, Nature 438, 65 (2005).

${ }^{8}$ Y. Okawachi, M. S. Bigelow, J. E. Sharping, Z. Zhu, A. Schweinsberg, D. J. Gauthier, R. W. Boyd, and A. L. Gaeta, Phys. Rev. Lett. 94, 153902 (2005).

${ }^{9}$ C. Liu, Z. Dutton, C. H. Behroozi, and L. V. Hau, Nature 409, 490 (2001).
${ }^{10}$ D. Phillips, A. Fleischhauer, A. Mair, R. Walsworth, and M. D. Lukin, Phys. Rev. Lett. 86, 783 (2001).

${ }^{11}$ T. Itoh, P. Lavallard, J. Reydellet, and C. Benoit, Sol. State Commun. 37, 925 (1981).

${ }^{12}$ S. Chu and S. Wong, Phys. Rev. Lett. 48, 738 (1982).

${ }^{13}$ M. Kuwata, T. Kuga, H. Akiyama, T. Hirano, and M. Matsuoka, Phys. Rev. Lett. 61, 1226 (1988).

${ }^{14}$ T. V. Shubina, M. M. Glazov, A. A. Toropov, N. A. Gippius, A. Vasson, J. Leymarie, A. Kavokin, A. Usui, J. P. Bergman, G. Pozina, and B. Monemar, Phys. Rev. Lett. 100, 087402 (2008).

${ }^{15}$ T. Godde, I. A. Akimov, D. R. Yakovlev, H. Mariette, and M. Bayer, Phys. Rev. B 82, 115332 (2010).

${ }^{16}$ K. Ogawa, T. Katsuyama, and H. Nakamura, Phys. Rev. Lett. 64, 796 (1990).

${ }^{17}$ J. Lagois, Phys. Rev. B 23, 5511 (1981).

${ }^{18}$ G. Blattner, G. Kurtze, G. Schmieder, and C. Klingshirn, Phys. Rev. B 25, 7413 (1994). 
${ }^{19}$ S. F. Chichibu, T. Sota, G. Cantwell, D. B. Eason, and C. W. Litton, Jap. J. Appl. Phys. 93, 756 (2003).

${ }^{20}$ M. Zamfirescu, A. Kavokin, B. Gil, G. Malpuech, and M. Kaliteevski, Phys. Rev. B 65, 161205 (2001).

${ }^{21}$ G. Xiong, J. Wilinson, K. B. Ucer, and R. T. Williams, J. Phys. Condens. Matter 17, 7287 (2005).

${ }^{22}$ D. G. Thomas, J. Phys. Chem. 15, 86 (1960).

${ }^{23}$ W. R. L. Lambrecht, A. V. Rodina, S. Limpijumnong, B. Segall, and B. K. Meyer, Phys. Rev. B 65, 075207 (2004).

${ }^{24}$ C. Klingshirn, Phys. Stat. Sol. (b) 244, 3027 (2007).

${ }^{25}$ M. Cobet, C. Cobet, M. R. Wagner, N. Esser, C. Thomsen, and A. Hoffmann, Appl. Phys. Lett. 96, 031904 (2010).

${ }^{26}$ B. K. Meyer, H. Alves, D. M. Hofmann, W. Kriegseis, D. Forster, F. Bertram, J. Christen, A. Hoffmann, M. Straßburg, M. Dworzak,
U. Haboeck, and A. V. Rodina, Phys. Stat. Sol. (b) 241, 231 (2004).

${ }^{27}$ E. L. Ivchenko, Optical Spectroscopy of Semiconductor Nanostructures (Alpha Science, Harrow, UK, 2005), p. 627.

${ }^{28}$ Y. Gu, I. L. Kuskovsky, M. Yin, S. O'Brien, and G. F. Neumark, Appl. Phys. Lett. 85, 3833 (2004).

${ }^{29}$ S. Shokhovets, O. Ambacher, B. K. Meyer, and G. Gobsch, Phys. Rev. B 78, 035207 (2008).

${ }^{30}$ X. J. Wang, L. S. Vlasenko, S. J. Pearton, W. M. Chen, and I. A. Buyanova, J. Phys. D: Appl. Phys. 42, 175411 (2009).

${ }^{31}$ P.Y. Yu and M. Cardona, Fundamentals of Semiconductors (Springer-Verlag, Berlin, 1999), p. 620.

${ }^{32}$ C. Klingshirm, Semiconductor Optics (Springer-Verlag, Berlin, 2005), p. 797.

${ }^{33}$ Y. S. Park and J. R. Schneider, J. Appl. Phys. 39, 3049 (1968). 